\title{
ROLE OF CD166 IN MULTIPLE MYELOMA CELL HOMING TO THE BONE MARROW MICROENVIRONMENT AND DISEASE PROGRESSION
}

\author{
Linlin $\mathrm{Xu}^{1}$, Christophe Machal ${ }^{2}$, Hao $\mathrm{Wu}^{1}$, Bradley Poteat ${ }^{3}$, Colin $\mathrm{Crean}^{3}$, Angelo A. Cardoso ${ }^{3}$, John M. \\ Chirgwin $^{3}$, Helmut Hanenberg ${ }^{2}$, Khalid S. Mohammad ${ }^{3}$, Attaya Suvannasankha ${ }^{3,4}$, Edward F. Srour ${ }^{1,2,3}$ \\ ${ }^{1}$ Department of Microbiology and Immunology, Indiana University School of Medicine, Indianapolis, IN, USA \\ ${ }^{2}$ Department of Pediatrics, Indiana University School of Medicine, Indianapolis, IN, USA \\ ${ }^{3}$ Department of Medicine, Indiana University School of Medicine, Indianapolis, IN, USA \\ ${ }^{4}$ Richard L. Roudebush Veterans' Administration Hospital, Indianapolis, IN, USA
}

Multiple myeloma ( $\mathrm{MM}$ ) is a plasma cell malignancy characterized by multiple lytic lesions throughout the skeleton, suggesting that trafficking of MM cells from the bone marrow (BM) and lodgment of these cells at secondary sites is important in disease progression. CD38+CD138- MM cells were previously characterized as putative MM stem cells (MMSC, Cancer Res. 2008; 68(1):190-7.). We analyzed CD38+CD138- cells contained within the MM cell line $\mathrm{H} 929$ and determined that a fraction of these cells (29.9\% $\pm 1.4 \%)$ expresses CD166. CD166 is a member of the immunoglobulin superfamily capable of mediating both homophilic and heterophilic (CD6) interactions and has been shown to enhance metastasis and invasion in several tumors including breast cancer and melanoma. Studies from our laboratory suggest that CD38+CD138-CD166+ MM cells possess many functional properties commonly associated with MMSC including cell cycle quiescence, maintenance and propagation of daughter cells on a stromal substrate and gene expression profile. We hypothesized that CD166 promotes MM cell trafficking to the BM and is critical for disease progression. To test this hypothesis, H929-GFP myeloma cells were injected intravenously into NSG mice and GFP cells were recovered from the BM 14hr later. While only $3.3 \% \pm 1.5 \%$ of total H929-GFP cells express the CD38+CD138- phenotype, the frequency of CD38+CD138- cells contained in BM-homed H929-GFP cells was significantly higher $(53.4 \% \pm 3.7 \%, n=3$, $\mathrm{p}<0.01$ ), suggesting a preferential homing of MMSC to the marrow microenvironment. Interestingly, whereas only $29.9 \% \pm 1.4 \%$ of CD38+CD138- cells expressed CD166 prior to injection, $84.1 \% \pm 10.8 \%$ of BM-homed H929-GFP CD38+CD138- cells expressed CD166 ( $n=3, p<0.01)$, suggesting that CD166 plays a critical role in directing homing of MM cells to the BM. Next, CD166 expression on H929-GFP cells was knocked down (KD) with shRNA in order to examine if reduced CD166 expression inhibit the homing of MM cells to the BM. The number of BM-homed GFP cells was significantly decreased for CD166KD cells (5658 $\pm 904, n=6)$ compared to mock control $(8551 \pm 848, n=6 ; p<0.05)$. Interestingly, cells in which suppression of CD166 expression was not achieved with shRNA homed preferentially to the BM (4.3\% $\pm 0.3 \%$ CD166+cells in CD166 KD H929-GFP before injection versus $29.3 \% \pm 3.6 \%$ in BM-homed GFP cells). Then we compared the progression of MM in NSG mice initiated with mock control or CD166 KD H929-GFP cells. Disease progression in mice receiving control cells was more rapid compared to that in mice receiving CD166KD cells as evidenced by serum levels of human IgA (kappa) at 4 weeks posttransplantation $(240.5 \pm 67.1 \mathrm{ng} / \mathrm{ml}$ versus $45.1 \pm 33.0 \mathrm{ng} / \mathrm{ml}, \mathrm{n}=3 ; \mathrm{p}<0.05)$. We next examined the potential role of CD166 in osteolytic lesions using a novel Ex Vivo Organ Culture Assay (EVOCA) in which MM cells are co-cultured over calvariae from 10d-old pups for 7 days creating an in vitro 3D system for the 
interaction of MM cells with bone microenvironment. Data from EVOCA with H929 cells showed that bone osteolytic lesions are substantially reduced when CD166 is absent on either MM (CD166- fraction) or osteoblast lineage cells (calvariae from CD166 ${ }^{-/-}$mice). Furthermore, co-culturing CD166+ or CD166H929 cells with bone marrow stromal cells (BMSC) from WT or CD166 ${ }^{-1-}$ mice revealed that mRNA levels of receptor activator of NF-KB ligand (RANKL) are decreased when CD166 is absent on either MM or stromal cells while mRNA levels of osteoprotegerin (OPG), an important inhibitor of osteoclastogenesis, are not altered. This resulted in decreased RANKL/OPG ratios in cultures containing a CD166component suggesting reduced MM-induced osteoclastogenesis in the absence of CD166. Interestingly, levels of M-CSF and IL- 6 were similar in all these cultures suggesting that loss of CD166 may mediate suppression of osteolytic lesions through the downregulation of RANKL. Together, these results suggest that CD166 plays an important role in homing and retention of MM cells in the BM and promotes MM disease progression as well as bone-lytic disease and that CD166 may serve as a therapeutic target in the treatment of MM. 